\title{
Folding a Single-Molecule Junction ${ }^{\dagger}$
}

† Ms. Chuanli Wu, Mr. Demetris Bates, Dr. Nicoló Ferri, Mr. Aidan Thomas, Dr. Craig M. Robertson, Prof. Dr. Simon J. Higgins, Prof. Dr. Richard J. Nichols and Dr. Andrea Vezzoli

Department of Chemistry, University of Liverpool, Crown Street, Liverpool L69 7ZD, United Kingdom e-mail for Dr. Andrea Vezzoli: andrea.vezzoli@liverpool.ac.uk

Ms. Chuanli Wu

School of Chemistry and Materials Science, Nanjing Normal University, Nanjing 210023, People's Republic of China

Dr. Sara Sangtarash and Dr. Hatef Sadeghi

School of Engineering, University of Warwick, Coventry CV4 7AL, U.K.

e-mail for Dr. Hatef Sadeghi: hatef.sadeghi@warwick.ac.uk

\section{Dr. Andrea Vezzoli}

Stephenson Institute for Renewable Energy, University of Liverpool, Peach Street, Liverpool L69 7ZF, United Kingdom

\#: these authors contributed equally to this work. 
Chuanli $\mathrm{Wu}^{\ddagger}$, Demetris Bates ${ }^{\ddagger}$, Sara Sangtarash ${ }^{\ddagger}$, Nicoló Ferri, Aidan Thomas, Simon J. Higgins, Craig M. Robertson, Richard J. Nichols, Hatef Sadeghi* and Andrea Vezzoli*

\section{Contents}

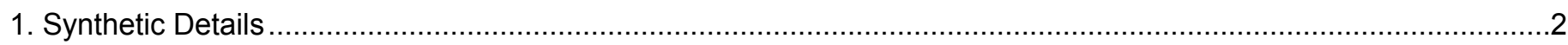

1.1 Synthesis of 1 .



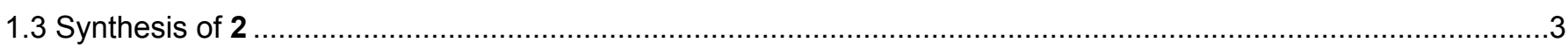

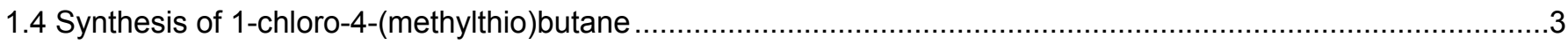

1.5 Synthesis of 3

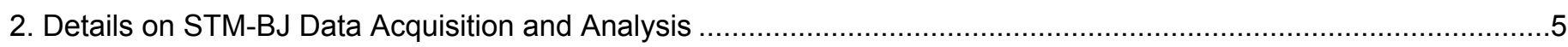



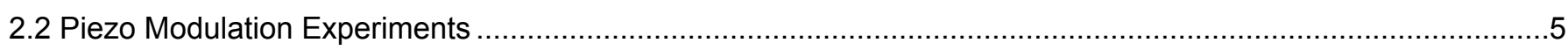

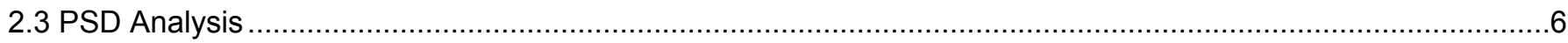

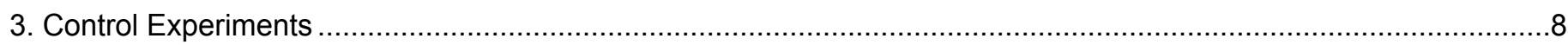

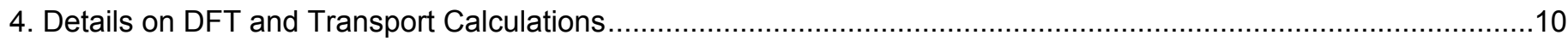

4.1 Molecule-Electrode Electron Transfer and Position of the LUMO ….......................................................



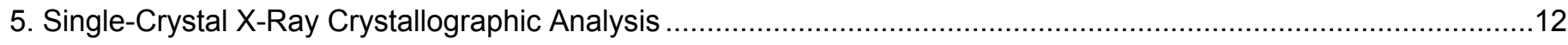

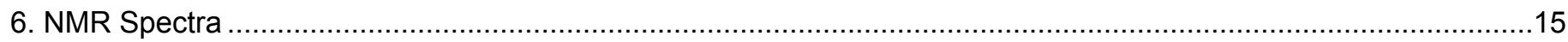



\section{Synthetic Details}

All reactions were carried out under an inert $\mathrm{N}_{2}$ atmosphere. THF and toluene were dried using an Innovative technology PS-400-6-MD solvent purification system. All other reagents and solvents were purchased from Sigma Aldrich, Acros Organics or Fischer and used as received. Column chromatography was performed on silica gel, monitored by thin layer chromatography (TLC) using Merck $60 \mathrm{~F}_{254}$ plates and visualised under UV light. NMR spectra for $\mathbf{1}$ and $\mathbf{2}$ were recorded on a Bruker Avance III HD and chemical shifts referenced to residual solvent or tetramethylsilane. NMR spectra for $\mathbf{3}$ were recorded on a Bruker Avance I and chemical shifts referenced to residual solvent or tetramethylsilane. Mass spectra were recorded by the University of Liverpool Analytical Services on an Agilent QTOF7200/QTOF6540 mass spectrometer. Elemental analysis was carried out by The University of Liverpool Analytical Services on an Elementar Vario Micro Cube. Fourier transform infrared (FT-IR) spectra were recorded with a PerkinElmer Spectrum 100 FT-IR spectrometer. 


\subsection{Synthesis of 1}

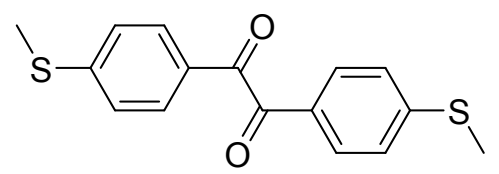

Based on a modified literature procedure. ${ }^{1}$ 4-bromothioanisole $(2.00 \mathrm{~g}, 9.85 \mathrm{mmol})$ was dissolved in anhydrous THF (50 mL) and cooled to $-78^{\circ} \mathrm{C}$. n-Butyllithium $(2.5 \mathrm{M}, 4.10 \mathrm{~mL})$ was added dropwise and the resulting white suspension (A) was stirred for $1 \mathrm{~h}$. To a second flask was added copper (I) bromide (1.41 g, $9.83 \mathrm{mmol})$, anhydrous lithium bromide $(1.71 \mathrm{~g}, 19.69 \mathrm{mmol})$ and anhydrous THF $(30 \mathrm{~mL})$, and the mixture (B) was stirred for $10 \mathrm{~min}$ before being cooled to $-78^{\circ} \mathrm{C}$. The organolithium solution $\mathrm{A}$ was added dropwise to $B$ and the mixture was warmed to RT for 5 min before again being cooled to $-78^{\circ} \mathrm{C}$, resulting in a yellow solution. To a third flask was added oxalyl chloride $(0.41 \mathrm{~mL}, 4.78 \mathrm{mmol})$ and anhydrous THF $(70 \mathrm{~mL})$. This solution was cooled to $-78{ }^{\circ} \mathrm{C}$ and the yellow solution $(A+B)$ was added dropwise, and stirred for $3 \mathrm{hr}$. The resulting yellow solution was then warmed to $\mathrm{RT}$ and quenched with sat. $\mathrm{NH}_{4} \mathrm{Cl}$ solution $(30 \mathrm{~mL})$. The mixture was partitioned between water $(40 \mathrm{~mL})$ and ethyl acetate $(40 \mathrm{~mL})$ and the organic layer washed with water $(3 \times 40 \mathrm{~mL})$ and brine $(40 \mathrm{~mL})$. All aqueous washings were combined, extracted with $\mathrm{CH}_{2} \mathrm{Cl}_{2}(3 \times 40 \mathrm{~mL})$ and the $\mathrm{CH}_{2} \mathrm{Cl}_{2}$ extracts washed with brine $(40 \mathrm{~mL})$. All organic extracts were combined, dried over anhydrous $\mathrm{MgSO}_{4}$ and the solvent removed under reduced pressure. Yellow needles of pure product were obtained by recrystallisation from ethyl acetate (0.49 g, $34 \%)$ : $\mathrm{mp}$ : 151-153 ${ }^{\circ} \mathrm{C}$; IR $\left(\mathrm{cm}^{-1}\right)$ : 3286, 3007, 2926, 1648, 1543, 1552; ${ }^{1} \mathrm{H}$ NMR ( $\left.\mathrm{CDCl}_{3}, \mathrm{TMS}, 400 \mathrm{MHz}\right): \delta=7.86$ (d, $\left.4 \mathrm{H}, J=8.4 \mathrm{~Hz}\right), 7.29(\mathrm{~d}, 4 \mathrm{H}, J=8.4 \mathrm{~Hz}), 2.53(\mathrm{~s}, 6 \mathrm{H})$ ppm; ${ }^{13} \mathrm{C}\left\{{ }^{1} \mathrm{H}\right\} \operatorname{NMR}\left(\mathrm{CDCl}_{3}, 100 \mathrm{MHz}\right): \delta=193.5,148.8,130.2,129.3,125.1,14.6$ ppm; Calc. for $\mathrm{C}_{16} \mathrm{H}_{14} \mathrm{O}_{2} \mathrm{~S}_{2}$ : C, 63.55; H, 4.67; S, 21.20. Found: C, 63.44; H, 4.76; S, 20.94. MS $\left(\mathrm{Cl}^{+}\right): \mathrm{m} / \mathrm{z} 303.1[\mathrm{M}+\mathrm{H}]^{+}$, calc. for $\mathrm{C}_{16} \mathrm{H}_{15} \mathrm{O}_{2} \mathrm{~S}_{2} 303.1$. 
1.2 Synthesis of 4-(methylthio)benzyltriphenylphosphonium bromide<smiles>CSc1ccc(CP(Br)Br)cc1</smiles>

Based on a modified literature procedure. ${ }^{2}$ Triphenylphosphine $(0.49 \mathrm{~g}, 1.87 \mathrm{mmol})$ was dissolved in anhydrous toluene $(5 \mathrm{ml})$ and heated to reflux. A solution of (4-methylthio)benzyl bromide $(0.40 \mathrm{~g}, 1.84 \mathrm{mmol})$ in anhydrous toluene $(5 \mathrm{ml})$ was prepared and added to the refluxing solution. The reaction was cooled after 18 hours and the crude product was collected by filtration. Recrystallisation from $\mathrm{CH}_{2} \mathrm{Cl}_{2} /$ hexane yielded the pure product as a white crystalline solid $(0.68 \mathrm{~g}, 77 \%):{ }^{1} \mathrm{H} \mathrm{NMR}\left(\mathrm{CDCl}_{3}, \mathrm{TMS}, 400 \mathrm{MHz}\right): \delta=7.80-7.70(\mathrm{~m}$, $9 \mathrm{H}), 7.67-7.59(\mathrm{~m}, 6 \mathrm{H}), 7.07-7.02\left(\mathrm{dd}, 2 \mathrm{H}, \mathrm{J}^{\mathrm{HH}}=8.5 \mathrm{~Hz}, \mathrm{JHP}^{\mathrm{HP}}=2.4 \mathrm{~Hz}\right), 6.96\left(\mathrm{~d}, 2 \mathrm{H}, \mathrm{J}^{\mathrm{HH}}=8.1 \mathrm{~Hz}\right), 5.37(\mathrm{~d}$, $2 \mathrm{H}, \mathrm{JHP}=14.3 \mathrm{~Hz}), 2.39(\mathrm{~s}, 3 \mathrm{H}) \mathrm{ppm} ;{ }^{13} \mathrm{C}\left\{{ }^{1} \mathrm{H}\right\} \mathrm{NMR}\left(\mathrm{CDCl}_{3}, 100 \mathrm{MHz}\right): \delta=139.2(\mathrm{~d}, J \mathrm{cP}=4.5 \mathrm{~Hz}), 135.0(\mathrm{~d}$, $J \mathrm{CP}=2.9 \mathrm{~Hz}), 134.4(\mathrm{~d}, \mathrm{JCP}=9.7 \mathrm{~Hz}), 131.9(\mathrm{~d}, \mathrm{JCP}=5.7 \mathrm{~Hz}), 130.1(\mathrm{~d}, J \mathrm{CP}=12.4 \mathrm{~Hz}), 126.2(\mathrm{~d}, J \mathrm{CP}=3.2$ $\mathrm{Hz}), 123.4(\mathrm{~d}, \mathrm{JCP}=8.9 \mathrm{~Hz}), 117.8(\mathrm{~d}, \mathrm{JCP}=85.5 \mathrm{~Hz}), 30.3(\mathrm{~d}, \mathrm{JCP}=46.7 \mathrm{~Hz}), 15.3 \mathrm{ppm} ;{ }^{31} \mathrm{P}\left\{{ }^{1} \mathrm{H}\right\} \mathrm{NMR}(162$ $\left.\mathrm{MHz}, \mathrm{CDCl}_{3}\right): \delta=22.6 \mathrm{ppm}$; HRMS $\left(\mathrm{ESI}^{+}\right): \mathrm{m} / \mathrm{z} 399.1332[\mathrm{M}]^{+}$, calc. for $\mathrm{C}_{26} \mathrm{H}_{24} \mathrm{PS} 399.1331$.

\subsection{Synthesis of 2}

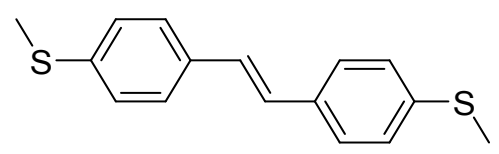

Based on a literature procedure. ${ }^{2}$ Pure 2 was obtained as a white powder $(0.04 \mathrm{~g}, 14 \%):{ }^{1} \mathrm{H}$ NMR $\left(\mathrm{CDCl}_{3}\right.$, TMS, $400 \mathrm{MHz}): \delta=7.42(\mathrm{~d}, 4 \mathrm{H}, J=8.4 \mathrm{~Hz}), 7.23(\mathrm{~d}, 4 \mathrm{H}, J=8.4 \mathrm{~Hz}), 7.01(\mathrm{~s}, 2 \mathrm{H}), 2.50(\mathrm{~s}, 6 \mathrm{H}) \mathrm{ppm} ;{ }^{13} \mathrm{C}\left\{{ }^{1} \mathrm{H}\right\}$ $\operatorname{NMR}\left(\mathrm{CDCl}_{3}, 100 \mathrm{MHz}\right): \delta=137.8,134.3,127.4,126.8,126.7,15.9$ ppm; HRMS (Cl+, CH4): m/z 273.0758 $[\mathrm{M}+\mathrm{H}]+$, calc. for $\mathrm{C}_{16} \mathrm{H}_{17} \mathrm{~S}_{2} 273.0766$.

1.4 Synthesis of 1-chloro-4-(methylthio)butane<smiles>CSCCCCCl</smiles>

Based on a literature procedure. ${ }^{3}$ Pure 1-chloro-4-(methylthio)butane was obtained as a clear oil (1.41 g, 59 \%): ${ }^{1} \mathrm{H}$ NMR $\left(\mathrm{CDCl}_{3}, \mathrm{TMS}, 400 \mathrm{MHz}\right): \delta=3.59(\mathrm{t}, 2 \mathrm{H}, J=6.5 \mathrm{~Hz}), 2.55(\mathrm{t}, 2 \mathrm{H}, J=7.1 \mathrm{~Hz}), 2.12(\mathrm{~s}, 3 \mathrm{H}), 1.95-$ $1.87(\mathrm{~m}, 2 \mathrm{H}), 1.83-1.74(\mathrm{~m}, 2 \mathrm{H}) \mathrm{ppm} ;{ }^{13} \mathrm{C}\left\{{ }^{1} \mathrm{H}\right\} \mathrm{NMR}\left(\mathrm{CDCl}_{3}, 100 \mathrm{MHz}\right): \delta=44.6,33.4,31.4,26.2,15.4 \mathrm{ppm}$. 
1.5 Synthesis of 3<smiles>CSCCCCC(=O)c1ccc(SC)cc1</smiles>

Magnesium turnings $(0.73 \mathrm{~g}, 30.03 \mathrm{mmol})$ and a few crystals of iodine were suspended in anhydrous THF $(20 \mathrm{~mL})$. A portion of a solution of 1-chloro-4-(methylthio)butane $(0.89 \mathrm{~g}, 6.42 \mathrm{mmol})$ in anhydrous THF (20 $\mathrm{mL}$ ) was added, the reaction activated and the remaining solution added dropwise. The mixture was heated at reflux for 4 hours and then cooled to RT. A solution of 4-(methylthio)benzoyl chloride $(1.44 \mathrm{~g}, 7.71 \mathrm{mmol}$ ) in anhydrous THF (30 mL) was prepared, cooled to $-78^{\circ} \mathrm{C}$ and the Grignard reagent was added dropwise to this solution. The cloudy mixture was stirred at $-78{ }^{\circ} \mathrm{C}$ for $1 \mathrm{hr}$, warmed to RT over 20 mins and quenched with sat. $\mathrm{NH}_{4} \mathrm{Cl}$ solution $(20 \mathrm{~mL})$. The mixture was partitioned between ethyl acetate $(25 \mathrm{~mL})$ and water and the aqueous layer extracted with ethyl acetate $(4 \times 25 \mathrm{~mL})$. The combined organics were washed with water $(3 \times 50 \mathrm{~mL})$ and brine $(45 \mathrm{~mL})$, dried over $\mathrm{MgSO}_{4}$ and the solvent removed under reduced pressure leaving a yellow oil. The material was purified by column chromatography ( $8: 1$ petroleum ether/ethyl acetate) yielding pure 3 as a clear oil which crystallised on standing to a white solid. A second crop of pure crystalline needles was obtained by recrystallisation from hexane of the impure, product-containing fractions, (total yield -0.27


TMS, $400 \mathrm{MHz}$ ): $\delta=7.86$ (d, 2H, $J=8.4 \mathrm{~Hz}$ ), 7.25 (d, 2H, $J=8.4 \mathrm{~Hz}), 2.94(\mathrm{t}, 2 \mathrm{H}, J=7.2 \mathrm{~Hz}), 2.53 / 2.51$ (t/s - overlapping, $5 \mathrm{H}, J=7.3 \mathrm{~Hz}), 2.09(\mathrm{~s}, 3 \mathrm{H}), 1.87-1.79(\mathrm{~m}, 2 \mathrm{H}), 1.72-1.64(\mathrm{~m}, 2 \mathrm{H}) \mathrm{ppm} ;{ }^{13} \mathrm{C}\left\{{ }^{1} \mathrm{H}\right\} \mathrm{NMR}\left(\mathrm{CDCl}_{3}\right.$, $100 \mathrm{MHz}): \delta=199.0,145.7,133.3,128.5,125.0,37.8,34.0,28.8,23.5,15.5,14.8$ ppm; Calc. for $\mathrm{C}_{13} \mathrm{H}_{18} \mathrm{OS}_{2}$ : $\mathrm{C}=61.38 ; \mathrm{H}=7.13 ; \mathrm{S}=25.20 \%$. Found $\mathrm{C}=60.69 ; \mathrm{H}=6.90 ; \mathrm{S}=25.32 \%$. HRMS $\left(\mathrm{ESI} \mathrm{I}^{+}\right): \mathrm{m} / \mathrm{z} 277.0693$ $[\mathrm{M}+\mathrm{Na}]^{+}$, calc. for $\mathrm{C}_{13} \mathrm{H}_{18} \mathrm{OS}_{2} \mathrm{Na} 277.0691$. 


\section{Details on STM-BJ Data Acquisition and Analysis}

An STM (Keysight Technologies 5500 SPM) equipped with a freshly cut Au tip (Goodfellow Cambridge Au 99.99+\%) and a gold-on-mica substrate (200 nm Advent Research Materials Au 99.95\% thermally evaporated with an Edwards Auto306 on freshly cleaved on Agar Scientific muscovite mica) was used to fabricate and characterize the molecular junctions. The STM tip position and the tip-substrate bias were controlled by a function generator (Keysight Technologies 33522B) through the STM break-out box (Keysight Technologies N9447A). Measurements were either performed monitoring the current as a function of tip displacement with a home-built 4-channel preamplifier (low-bandwidth, based on the design originally developed by Meszaros et al. ${ }^{4}$ ), through a USB data acquisition board (NI-9215 - 16-bit @ $10 \mathrm{KSa} / \mathrm{s}$ ), or by monitoring the current as a function of time with a commercial single-channel transimpedance amplifier (Femto GmbH DLPCA-200), through a PXI system (National Instruments PXle-1062Q chassis, with a PXle4464 DAQ and a PXle-PCle8381 interface - 24-bit @ $100 \mathrm{kSa} / \mathrm{s}$ ). Data acquisition and processing is performed with bespoke software, developed in Labview and Python. In all experiments, the tip is driven into the substrate until the conductance is $>5 G_{0}$ to ensure the formation of a clean nano-contact, and then retracted with the desired voltage ramp applied to the piezo by more than $6 \mathrm{~nm}$. This ensures that the fabricated junctions are ruptured at the end of the withdrawal cycle. All experiments were performed at 200 $\mathrm{mV}$ tip-substrate bias (tip grounded), in a $1 \mathrm{mM}$ mesitylene solution of the target molecular wire. $>1000$ indentation cycles are performed prior to the introduction of the target solution in order to perform a mechanical annealing of the tip and ensure absence of contamination. For each experiment, $>5000$ individual traces are collected.

\subsection{Linear Piezo Ramp Experiments}

All $G_{z}$ traces collected with a linear piezo ramp $(20 \mathrm{~nm} / \mathrm{s}$ in this study) in the main text and later in this document were used to compile histograms and 2D plots with no further processing or selection.

\subsection{Piezo Modulation Experiments}

When a non-linear ramp was applied to the piezo to verify the mechanoresistive behavior, a modified protocol was applied. The raw traces, after conversion of current to conductance by using Ohm's law $(G=I / V)$ were sliced between abrupt stretches by analysing the second derivative of the signal applied to the piezo transducer and cutting when its value is above a threshold.

The slices were then fed into a sorting algorithm, that takes the average of the first and last modulation and checks that both are below $0.1 G_{0}$, above the noise level of the preamplifier $\left(10^{-5.5} G_{0}\right.$ at the bias voltage used in this study) and within the envelope of the gaussian fit of the conductance histogram peak. This process filters out slices where the tip and the substrate are in contact, those where there is no molecular bridge present and those where the molecular junction did not survive the whole modulation process, leaving only the slices relative to stable molecular junctions. All the slices selected by the sorting algorithm were then used to compile 2D density maps (conductance vs time). 

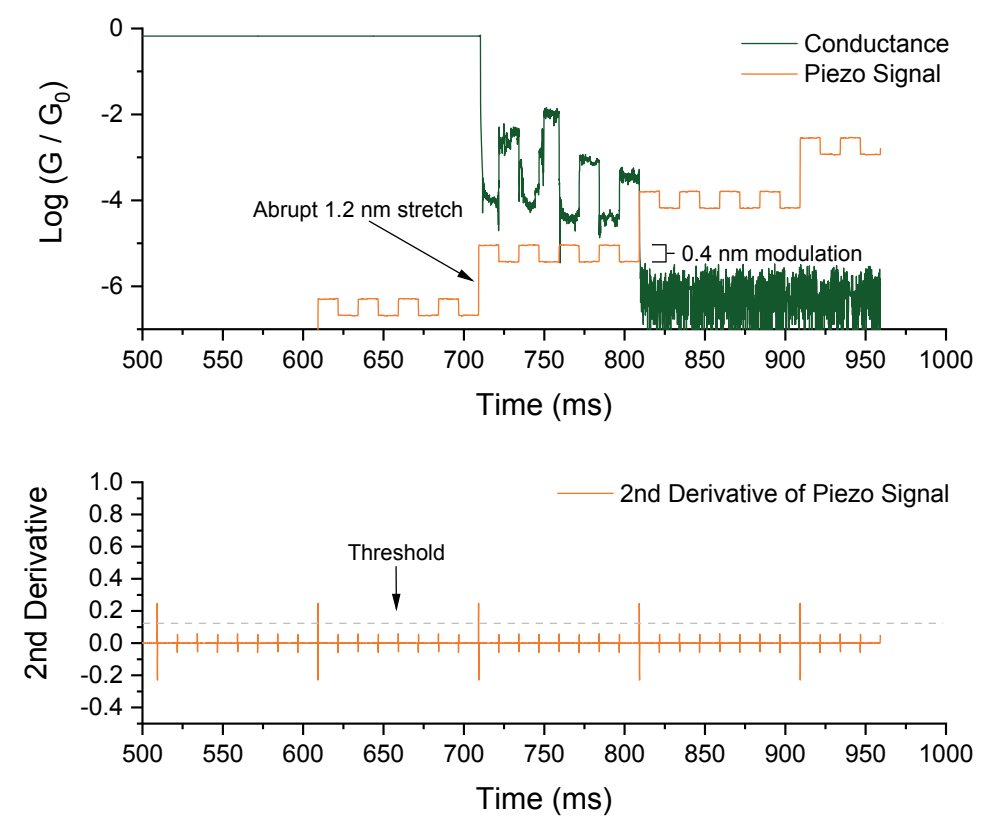

Figure S1: Example of piezo signal and conductance trace (top), and its second derivative (bottom) used in the slicing process.

More details on the process are available in our previous publication on the subject. ${ }^{5}$

\subsection{PSD Analysis}

In order to perform PSD analysis on the junction in both its compressed and relaxed state, we performed a measurement with a single modulation cycle, while monitoring current at $100 \mathrm{kSa} / \mathrm{s}$.

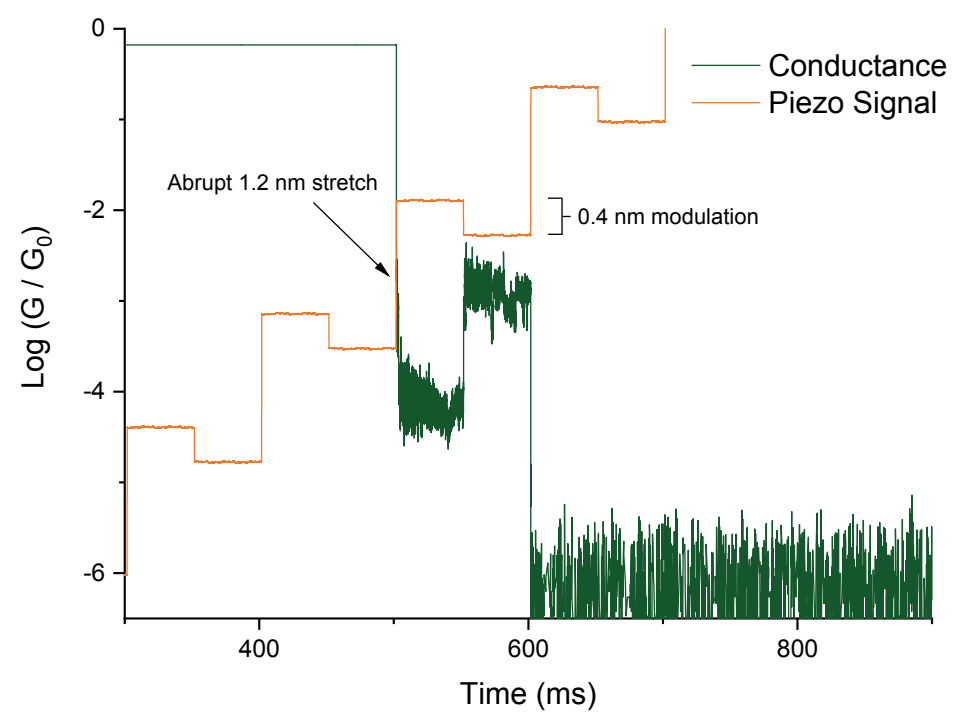

Figure S2: Example of piezo signal and conductance trace (top) for a single-modulation, high frequency data acquisition experiment.

The same algorithm described previously was used to slice and sort conductance traces. Note that the noise floor is higher in this high data acquisition speed experiment, as it scales with $\sqrt{\mathrm{Hz}}$. The individual, single compression cycle slices were separated between the compressed portion and the relaxed one, and each portion was fed to a FFT algorithm (NI Labview2019 Spectral Measurement) with no windowing. We selected 
3000 samples in each state to be fed to the FFT algorithm, from the middle of the region of interest, to reduce the influence of piezo creep and sample drift on the measured noise power.



Figure S3: Example single-modulation, high frequency conductance trace with the portion used for PSD analysis highlighted.

The FFT algorithm outputs the Power Spectral Density across the whole frequency spectrum. Numerical integration between $100 \mathrm{~Hz}$ and $1 \mathrm{kHz}$ yielded the Noise Power in this region, that was plotted vs $G_{A V G}$ (average of the conductance of the slice of interest) to give the plots shown in the main paper. The $1 \mathrm{kHz}$ and $100 \mathrm{~Hz}$ cut-offs were chosen as they have been demonstrated in the literature to be effective in limiting the contributions to Noise Power arising from thermal noise $(>1 \mathrm{kHz})$ and mechanical vibrations $(<100 \mathrm{~Hz}){ }^{6}$ 


\section{Control Experiments}

As discussed in the main text, we used $(E)$-1,2-bis(4-(methylthio)phenyl)ethene (2) to demonstrate that the switching phenomena do not occur in conformationally-locked compounds.

The synthesis of the compound is described earlier in this document. We started by measuring conductance using a linear ramp, in a regular STM-BJ experiment. No evidence of switching was found, as the results only show a single, sharp and well-resolved peak in the conductance histogram.
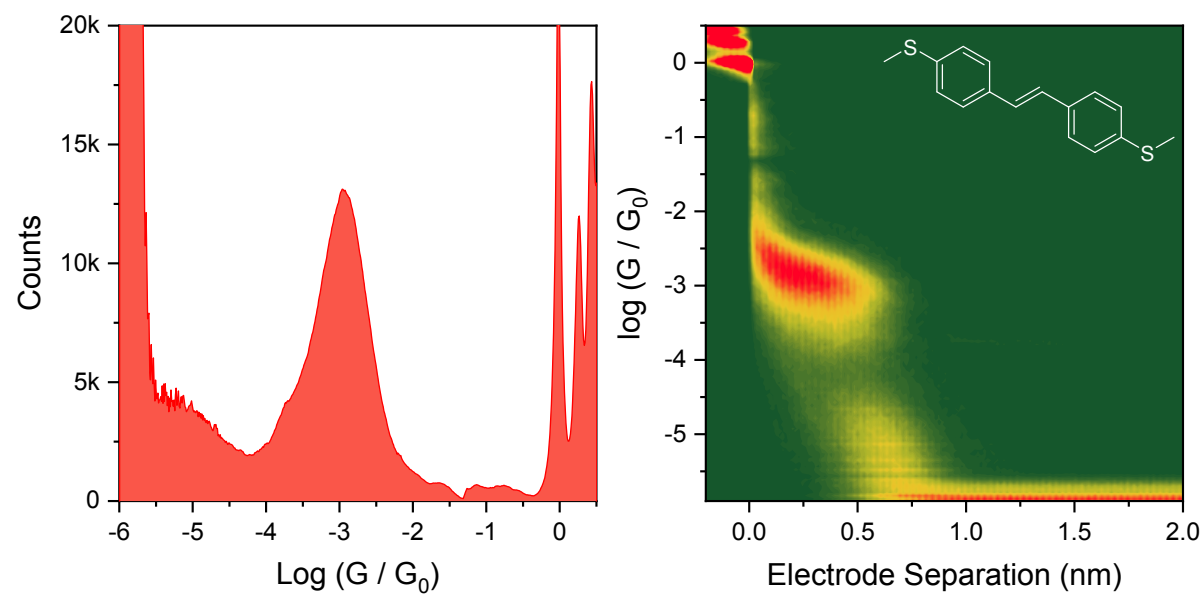

Figure S4: Conductance histogram (left) and 2D density map (right) for 2 (structure in inset). $5841 G_{Z}$ traces, $200 \mathrm{mV}$ tip-substrate bias.

Similarly, piezo-modulation experiments with the same ramp used for $\mathbf{1}$, made of a $1.2 \mathrm{~nm}$ abrupt stretch followed by $4 \times 0.4 \mathrm{~nm}$ modulations, showed only a very shallow increase of conductance (approximately twofold) in 2 upon junction compression (presented in the main text). We observed a similar behaviour in other thioanisole-terminated compounds ${ }^{5}$ and we attributed this to an increase in "lateral coupling" - weak interactions between the aromatic $\pi$-system and the metallic electrodes already observed in other molecular wires $^{7}$ and clearly not a change in the molecule-electrode binding mode.

Finally, we performed PSD analysis on the traces, to verify the transport pathway in 2 . No correlation between Noise Power / $G_{A V G}$ and $G_{A V G}$ was found, thus confirming the molecule has a pure through-bond charge transport mechanism, even under compression (Figure S5).


Figure S5: PSD analysis for $\mathbf{2}$ in the relaxed (left) and compressed (right) junction state. 
A second control experiment was performed on 5-(methylthio)-1-(4-(methylthio)phenyl)pentan-1-one (3). The synthesis of the compound is described earlier in this document.
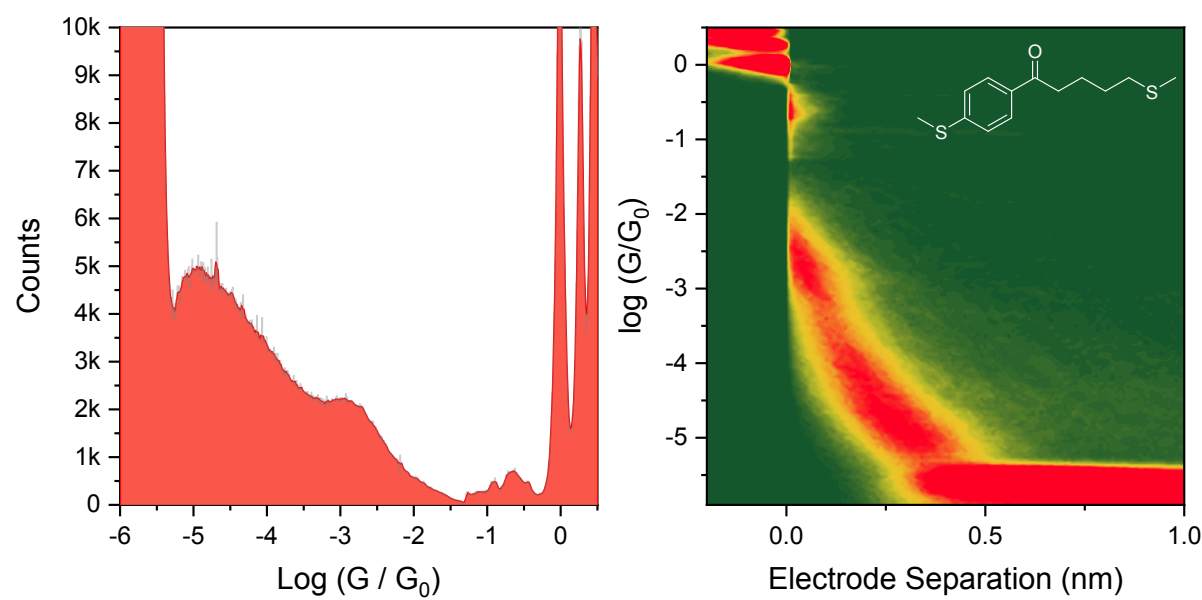

Figure S6: Conductance histogram (left) and 2D density map (right) for 3 (structure in inset). $4843 G_{z}$ traces, $200 \mathrm{mV}$ tip-substrate bias.

While linear-ramp STM-BJ experiments show that a small shoulder is indeed present at $\sim 10^{-3} \mathrm{G}_{0}$, therefore suggesting a switching mechanism could be in place, no high-conductance feature was accessible by mechanical compression. The piezo-modulation experiments (same ramp used for 1 and 2, $1.2 \mathrm{~nm}$ abrupt stretch followed by $4 \times 0.4 \mathrm{~nm}$ modulations presented in the main text) resulted in a rather noisy density map that evidences only a threefold increase in conductance, not comparable with that observed for compound 1 ( $21 \mathrm{x}$, see main text for a comparison plot).

PSD analysis showed again no change in charge transport pathway when the junction is compressed, as in both cases the noise power scales with values of $\mathrm{G}^{\mathrm{n}}$ intermediate between pure through-bond and pure through-space (respectively, $\sim G^{1.4}$ for the relaxed junction and $\sim G^{1.6}$ for the compressed junction).
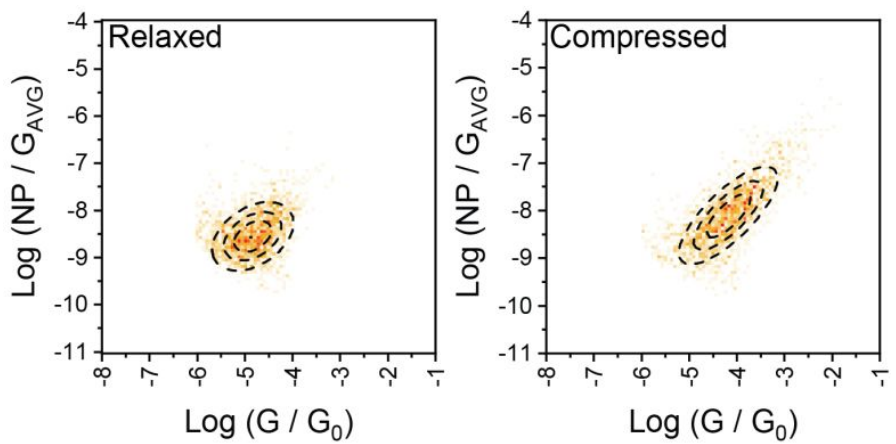

Figure S7: PSD analysis for 3 in the relaxed (left) and compressed (right) junction state. 


\section{Details on DFT and Transport Calculations}

The optimized geometry, ground state Hamiltonian and overlap matrix element of each structure was selfconsistently obtained using the SIESTA ${ }^{8}$ implementation of density functional theory (DFT). SIESTA employs norm-conserving pseudo-potentials to account for the core electrons, and linear combinations of atomic orbitals to construct the valence states. The generalized gradient approximation (GGA) of the exchange and correlation functional is used with the Perdew-Burke-Ernzerhof parameterization (PBE) a double- $\zeta$ polarized (DZP) basis set, a real-space grid defined with an equivalent energy cut-off of 250 Ry. The geometry optimization for each structure is performed to achieve forces smaller than $10 \mathrm{meV} / \AA$.

The mean-field Hamiltonian obtained from the converged DFT calculation was combined with Gollum ${ }^{9}$ implementation of the non-equilibrium Green's function method ${ }^{10}$ to calculate the phase-coherent, elastic scattering properties of each system consist of left gold (source) and right gold (drain) leads and the scattering region. The transmission coefficient $T(E)$ for electrons of energy $E$ (passing from the source to the drain) is calculated via the relation: $T(E)=\operatorname{Trace}\left(\Gamma_{R}(E) G^{R}(E) \Gamma_{L}(E) G^{R \dagger}(E)\right)$. In this expression, $\Gamma_{L, R}(E)=i$ $\left(\sum_{L, R}(E)-\sum_{L, R}{ }^{\dagger}(E)\right)$ describe the level broadening due to the coupling between left $(\mathrm{L})$ and right $(\mathrm{R})$ electrodes and the central scattering region, $\sum_{L, R}(E)$ are the retarded self-energies associated with this coupling and $G^{R}=$ $\left(E S-H-\sum_{L}-\sum_{R}\right)^{-1}$ is the retarded Green's function, where $H$ is the Hamiltonian and $S$ is overlap matrix. Using obtained transmission coefficient $T(E)$, the conductance could be calculated by Landauer formula $\left(G=G_{0}\right.$ $\left.\int d E T(E)(-\partial f / \partial E)\right)$ where $G_{0}=2 e^{2} / h$ is conductance quantum.

\subsection{Molecule-Electrode Electron Transfer and Position of the LUMO}

In the $T(E)$ curves shown in the main paper, the LUMO resonance of 1 appears to be pinned to the Fermi level of the electrodes. This arises from the strong electron-withdrawing effect of the diketone moiety that induces a strong electrode $\rightarrow$ molecule charge transfer phenomenon. We calculated the LUMO displacement (Figure S9) and net charge transfer (inset of Figure S9) as a function of the distance between the thiomethyl terminus of 1 and the electrode. As can be seen, up to 0.3 electrons are transferred at the optimal S-Au distance of $2.6 \AA$, and the LUMO is displaced by up to $20 \mathrm{meV}$ towards the electrodes $E_{F}$. 




Figure S8: LUMO displacement and net electron transfer (inset) as a function of the S-Au distance $d$ for molecule 1.

\subsection{Dihedral Torsional Barrier DFT Calculations}

In the manuscript we present MM2 Force Field modelling of the dihedral torsional barrier. The calculations was performed again using DFT. After optimization of the structure, a $\mathrm{Ph}-(\mathrm{C}=\mathrm{O})$ - fraction of the molecule was rotated to obtain the energy landscape.

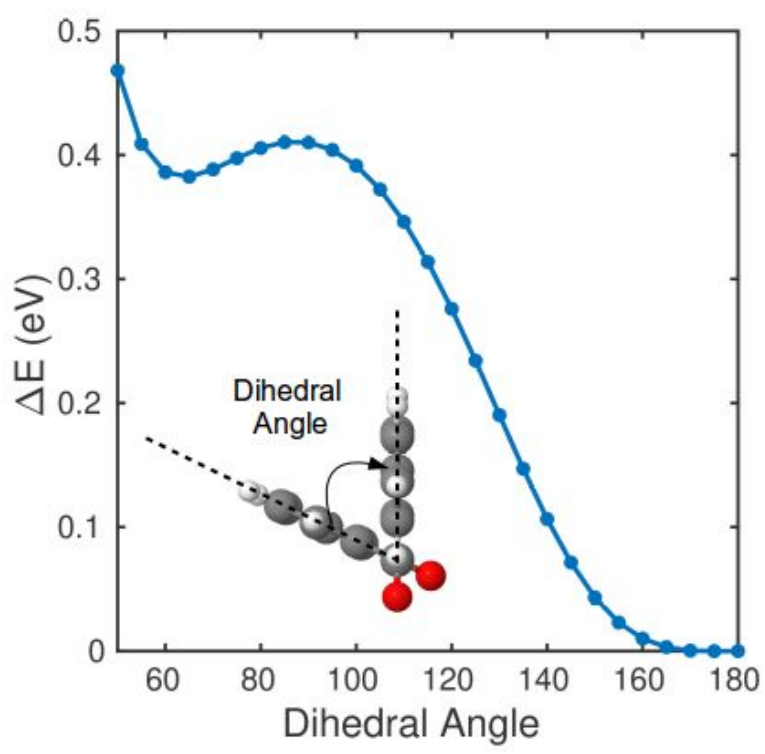

Figure S9: Energy landscape for the dihedral torsional barrier of benzil derivatives

As also described in the main text, DFT modelling gives a barrier height of $0.42 \mathrm{eV}$ for the anti $\rightleftarrows s y n$ interconversion, and a total energy difference between the two conformations of $0.38 \mathrm{eV}$. The values are comparable to those obtained with the MM2 Force Field computational method. 


\section{Single-Crystal X-Ray Crystallographic Analysis}

Yellow needles of 1 were grown by recrystallisation of the crude material from ethyl acetate $/ \mathrm{CH}_{2} \mathrm{Cl}_{2}$. Clear platelets of 2 were grown by dissolution in hot acetonitrile followed by evaporation. Crystals were mounted on a MiTeGen MicroMount using Parabar oil. X-ray data were collected at $150 \mathrm{~K}$ using Mo-Ka $(\lambda=0.71073)$ for 1 and Cu-Ka radiation ( $\lambda=1.54178 \AA$ ) for 2 on a Bruker Venture D8 dual-source micro-focus diffractometer fitted with a Photon 100 CMOS detector using $\varphi$ and $\omega$ scans. Integration and reduction were undertaken with Apex 3 software (Bruker AXS Inc.). Subsequent computations were employed using the OLEX2 software interface. ${ }^{11}$ Absorption corrections were applied to the data using SADABS (Bruker AXS Inc.). Structures were solved via direct methods using SHELXT ${ }^{12}$ followed by refinement with SHELXL. ${ }^{13}$ Non-hydrogen atoms were refined anisotropically.

\begin{tabular}{|c|c|c|}
\hline & 1 & 2 \\
\hline CCDC Number & 1950830 & 1988159 \\
\hline Empirical formula & $\mathrm{C}_{16} \mathrm{H}_{14} \mathrm{O}_{2} \mathrm{~S}_{2}$ & $\mathrm{C}_{16} \mathrm{H}_{16} \mathrm{~S}_{2}$ \\
\hline Formula weight & 302.39 & 272.41 \\
\hline Temperature/K & 150.0 & 150.02 \\
\hline Crystal system & Monoclinic & Monoclinic \\
\hline Space group & $\mathrm{C} 2 / \mathrm{c}$ & $P 2_{1} / c$ \\
\hline$a / \AA$ & $23.463(3)$ & $5.6118(2)$ \\
\hline b/Å & $4.0517(5)$ & $7.2767(3)$ \\
\hline$c / A$ & $15.524(2)$ & $33.6777(13)$ \\
\hline$\alpha /^{\circ}$ & 90 & 90 \\
\hline$\beta /^{\circ}$ & $106.312(6)$ & $93.1630(10)$ \\
\hline$y l^{\circ}$ & 90 & 90 \\
\hline Volume/ $\AA^{3}$ & $1416.3(3)$ & 1373.15(9) \\
\hline $\mathbf{Z}$ & 4 & 4 \\
\hline$\rho_{\text {calc }} \mathbf{g} / \mathrm{cm}^{3}$ & 1.418 & 1.318 \\
\hline$\mu / \mathrm{mm}^{-1}$ & 0.373 & 3.318 \\
\hline$F(000)$ & 632.0 & 576.0 \\
\hline Crystal size $/ \mathrm{mm}^{3}$ & $0.2 \times 0.08 \times 0.04$ & $0.2 \times 0.2 \times 0.06$ \\
\hline Radiation/ A & 0.71073 & 1.54178 \\
\hline $2 \Theta$ range for data collection $/^{\circ}$ & 5.468 to 52.858 & 5.256 to 133.378 \\
\hline Index ranges & $-28 \leq h \leq 28,-5 \leq k \leq 5,-19 \leq 1 \leq 19$ & $-6 \leq h \leq 6,-7 \leq k \leq 8,-39 \leq 1 \leq 40$ \\
\hline Reflections collected & 6486 & 16702 \\
\hline Independent reflections & $1440\left[R_{\text {int }}=0.0591, R_{\text {sigma }}=0.0332\right]$ & $2419\left[R_{\text {int }}=0.0282, R_{\text {sigma }}=0.0177\right]$ \\
\hline Data/restraints/parameters & $1440 / 0 / 92$ & $2419 / 0 / 165$ \\
\hline Goodness-of-fit on $F^{2}$ & 1.177 & 1.060 \\
\hline \multirow[t]{2}{*}{ Final $R$ indexes $[\mid>=2 \sigma(I)]$} & $R_{1}=0.0438$ & $R_{1}=0.0253$ \\
\hline & $\mathrm{w} R_{2}=0.1079$ & $\mathrm{w} R_{2}=0.0664$ \\
\hline \multirow[t]{2}{*}{ Final $R$ indexes [all data] } & $R_{1}=0.0539$ & $R_{1}=0.0286$ \\
\hline & $\mathrm{w} R_{2}=0.1137$ & $\mathrm{w} R_{2}=0.0683$ \\
\hline Largest diff. peak/hole / e $\AA^{-3}$ & $0.38 /-0.35$ & $0.22 /-0.20$ \\
\hline
\end{tabular}





Figure S11: Crystal structure of 2. Thermal ellipsoids are drawn at 50\% probability.

Colours used for crystallographic figures: carbon - grey, hydrogen - white, oxygen - red, sulphur - yellow. 


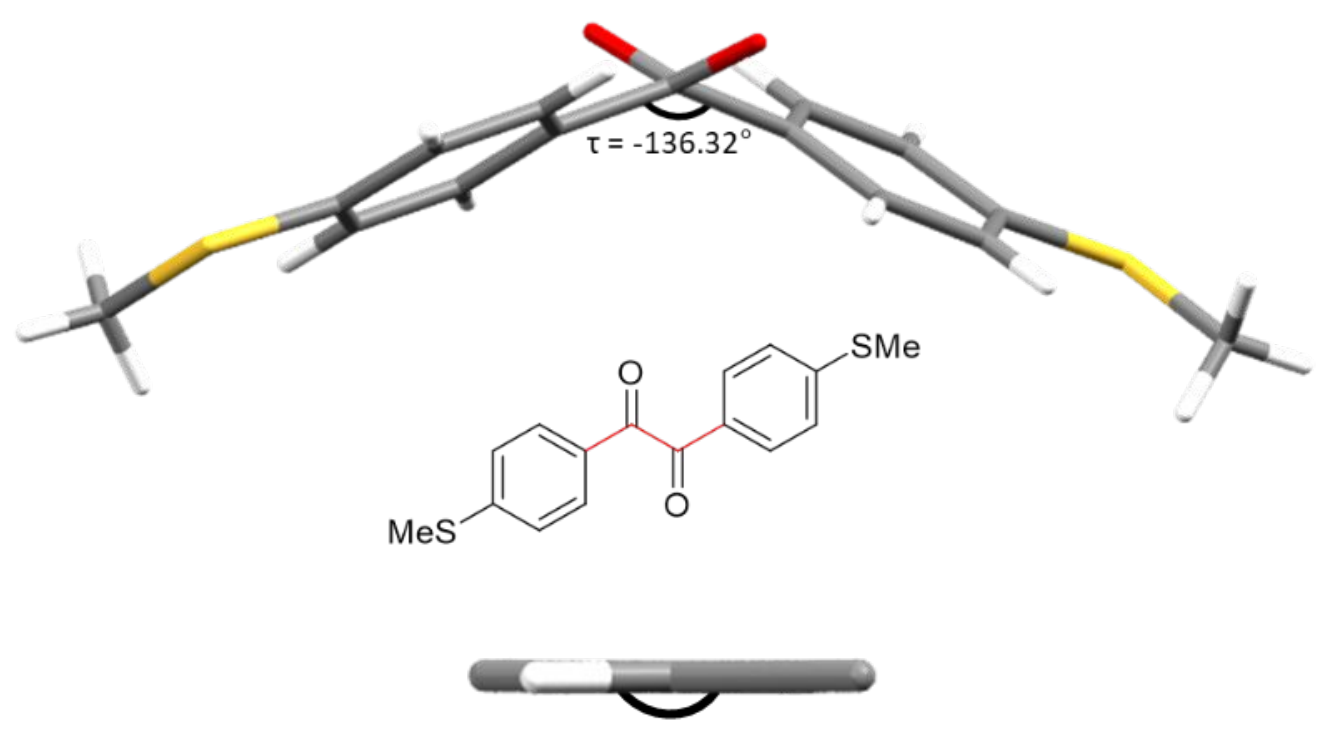

$\tau=-179.36^{\circ}$

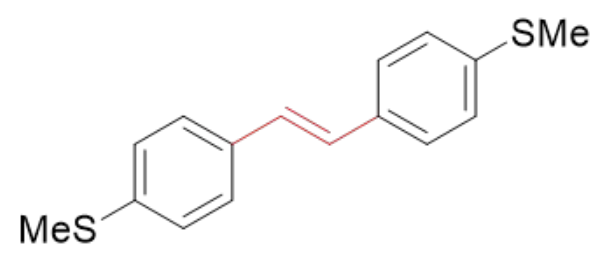

Figure S12: (top) View along central C-C bond of 1 with dihedral angle $(\tau)$ displayed. (bottom) View along central $\mathrm{C}=\mathrm{C}$ bond of $\mathbf{2}$ with $\tau$ displayed. Aromatic rings are omitted for clarity. $\tau$ is defined for the bonds highlighted in red for each structure. 


\section{NMR Spectra}

Submitted_by D Bates

Sample_name DB015 - dried

Lab_number 155

Staff/Student ID 201217734

DEPT2 CMR\#

Figure S13: ${ }^{1} \mathrm{H}$ NMR spectrum for 1.

Residual solvent peaks: 1.27: petroleum ether; 1.55 : water; 7.26 ppm: chloroform.

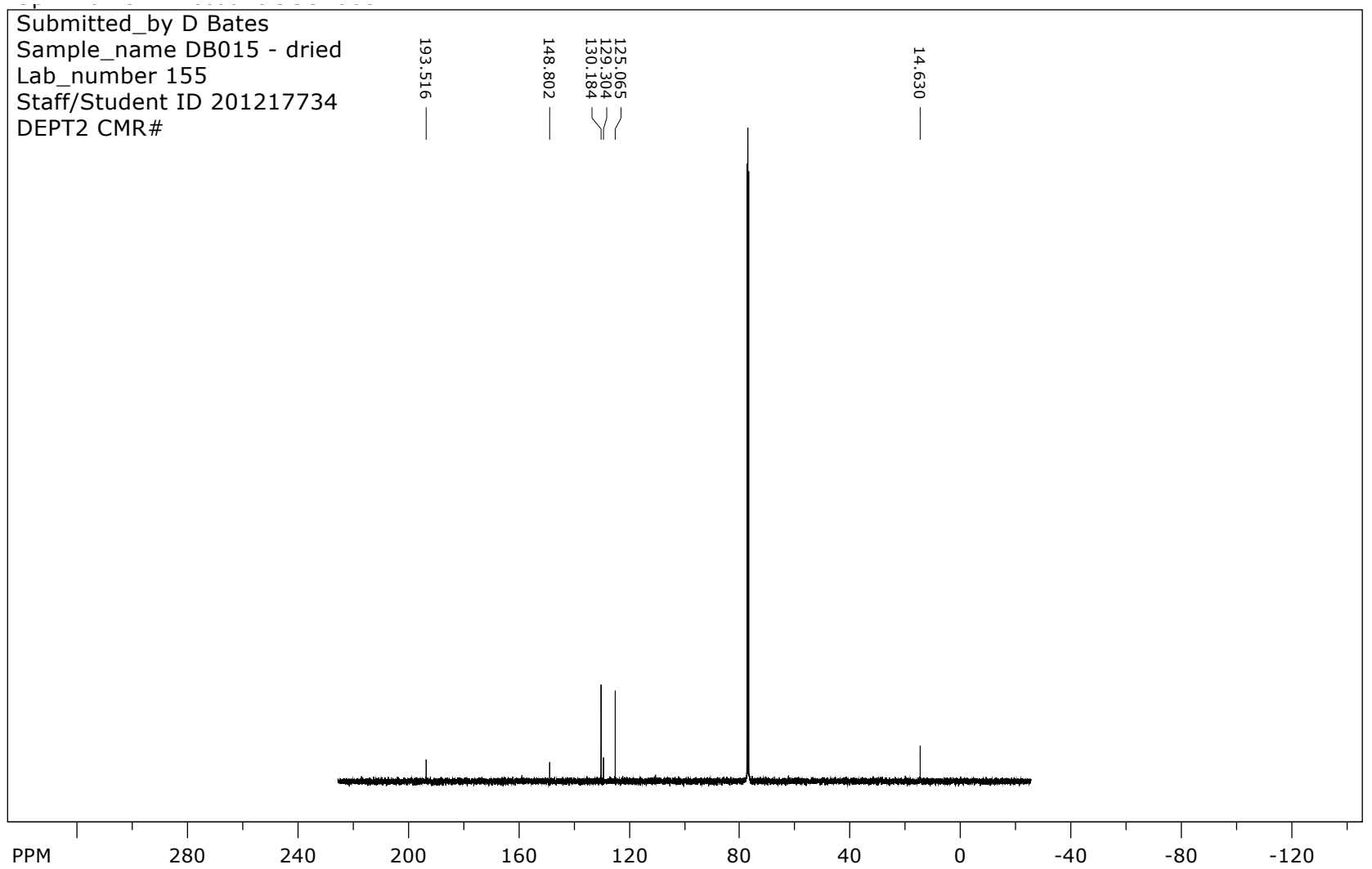

Figure S14: ${ }^{13} \mathrm{C}$ NMR spectrum for 1. 


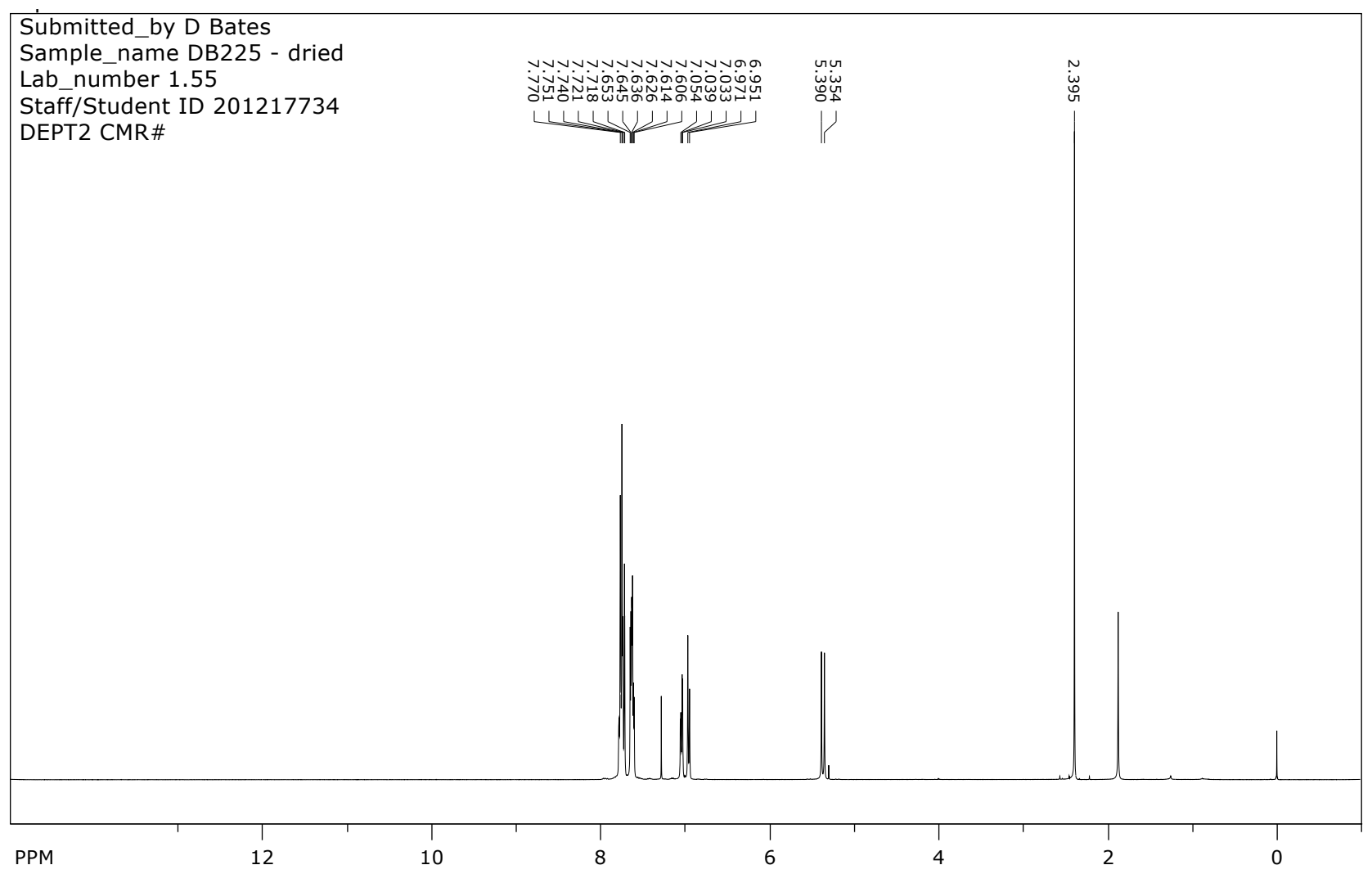

Figure S15: ${ }^{1} \mathrm{H}$ NMR spectrum for 4-(methylthio)benzyltriphenylphosphonium bromide. Residual solvent peaks: 1.88: water; $5.40: \mathrm{CH}_{2} \mathrm{Cl}_{2} ; 7.28$ ppm: chloroform.

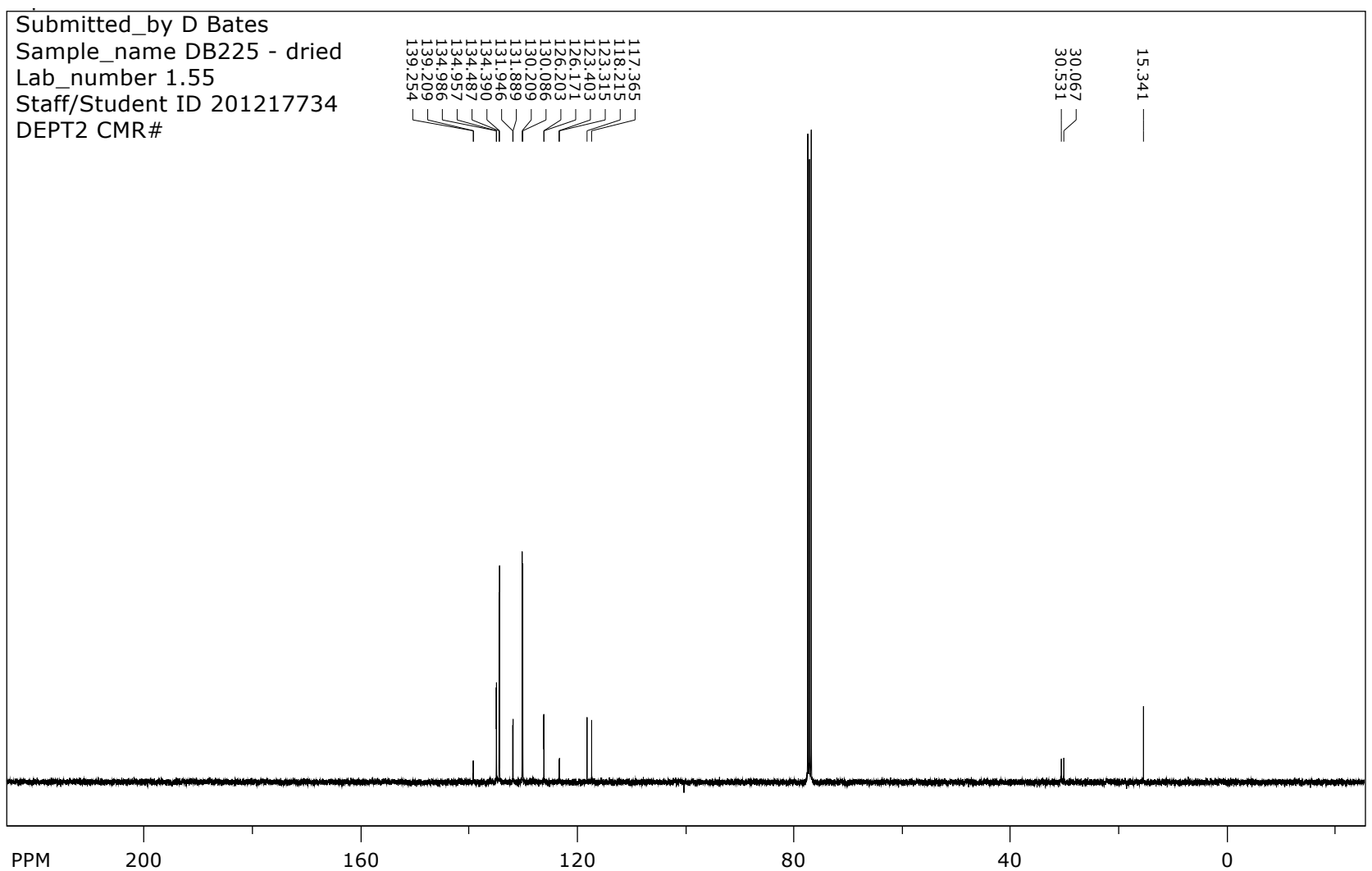

Figure S16: ${ }^{13} \mathrm{C}$ NMR spectrum for 4-(methylthio)benzyltriphenylphosphonium bromide. 


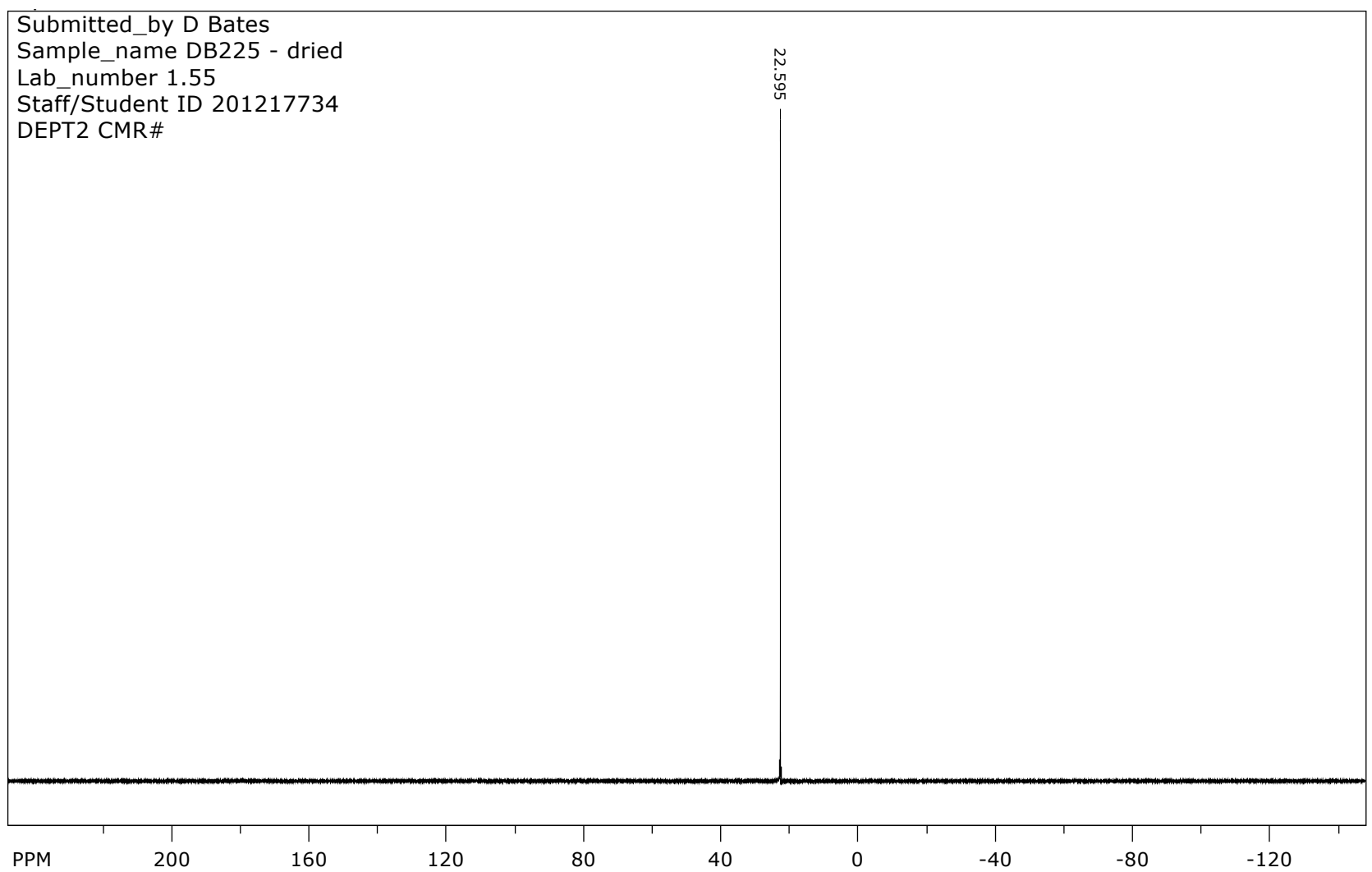

Figure S17 : ${ }^{31} \mathrm{P}$ NMR spectrum for 4-(methylthio)benzyltriphenylphosphonium bromide.

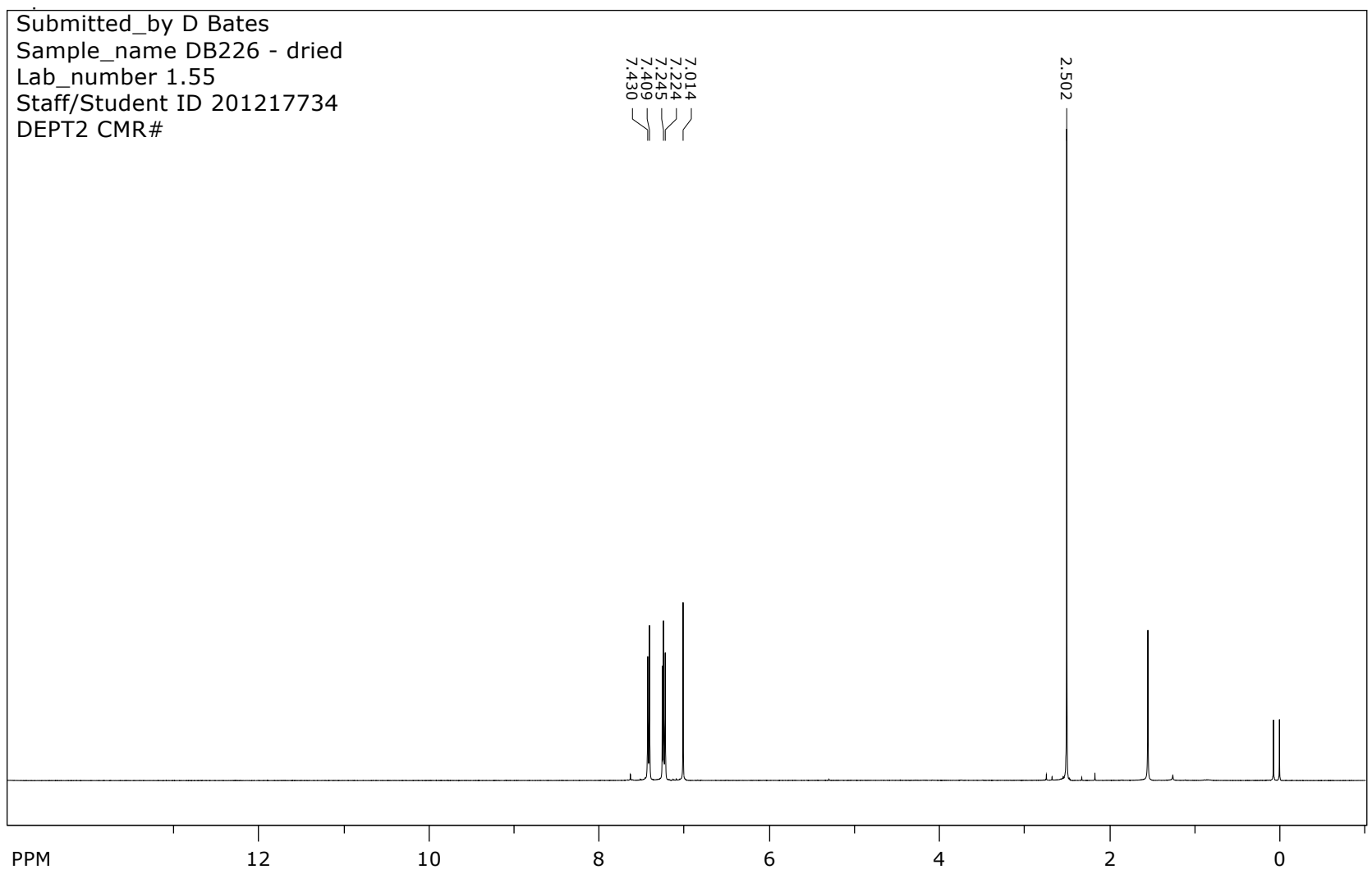

Figure S18: ${ }^{1} \mathrm{H}$ NMR spectrum for 2.

Residual solvent peaks: 0.07: silicon grease; 1.55 : water; 7.26 ppm: chloroform. 


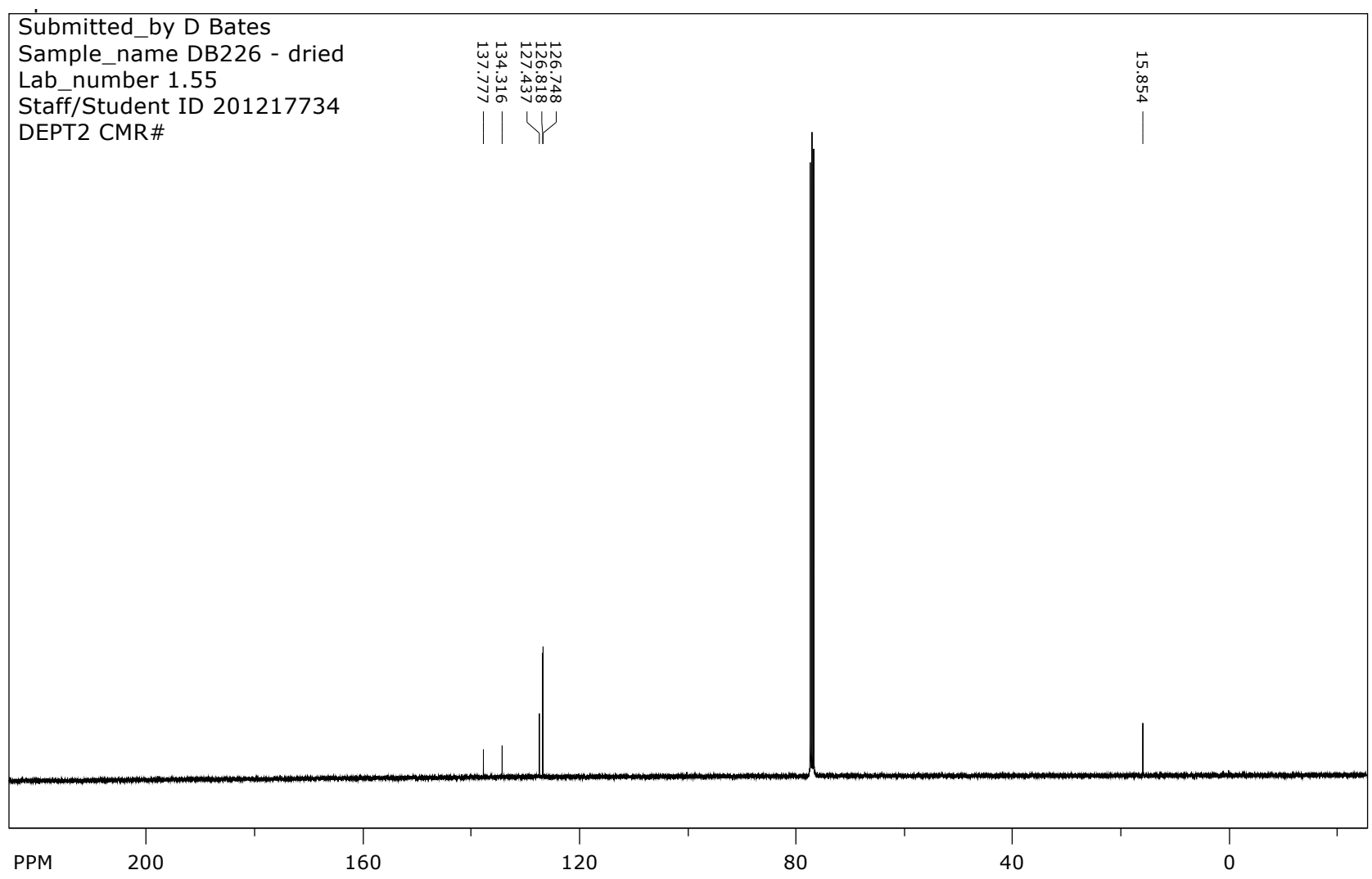

Figure S19: ${ }^{13} \mathrm{C}$ NMR spectrum for 2.

SpinWorks 4: DB235 - 1-chloro-4-(methylthio)butane

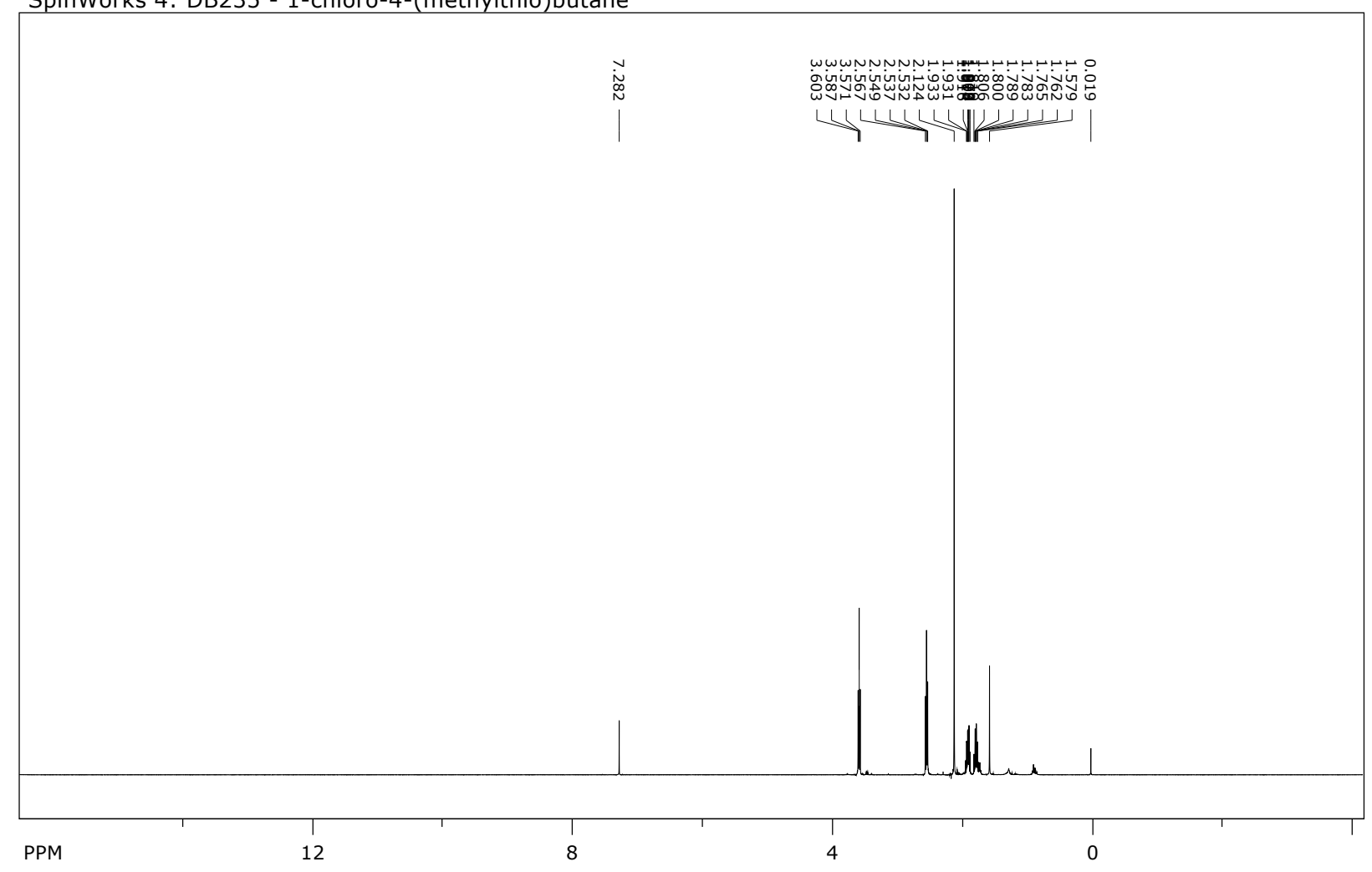

Figure S20 : ${ }^{1} \mathrm{H}$ NMR spectrum for 1-chloro-4-(methylthio)butane.

Residual solvent peaks: 1.58: water; 7.28 ppm: chloroform. 


\section{SUPPLEMENTARY INFORMATION}

SpinWorks 4: Account SCG10037

Submitted_by D Bates

Sample_name DB235 - 1-chloro-4-(methylthio)butane

Lab number 1.55

Staff/Student ID 201217734

DEPT2 CMR\#

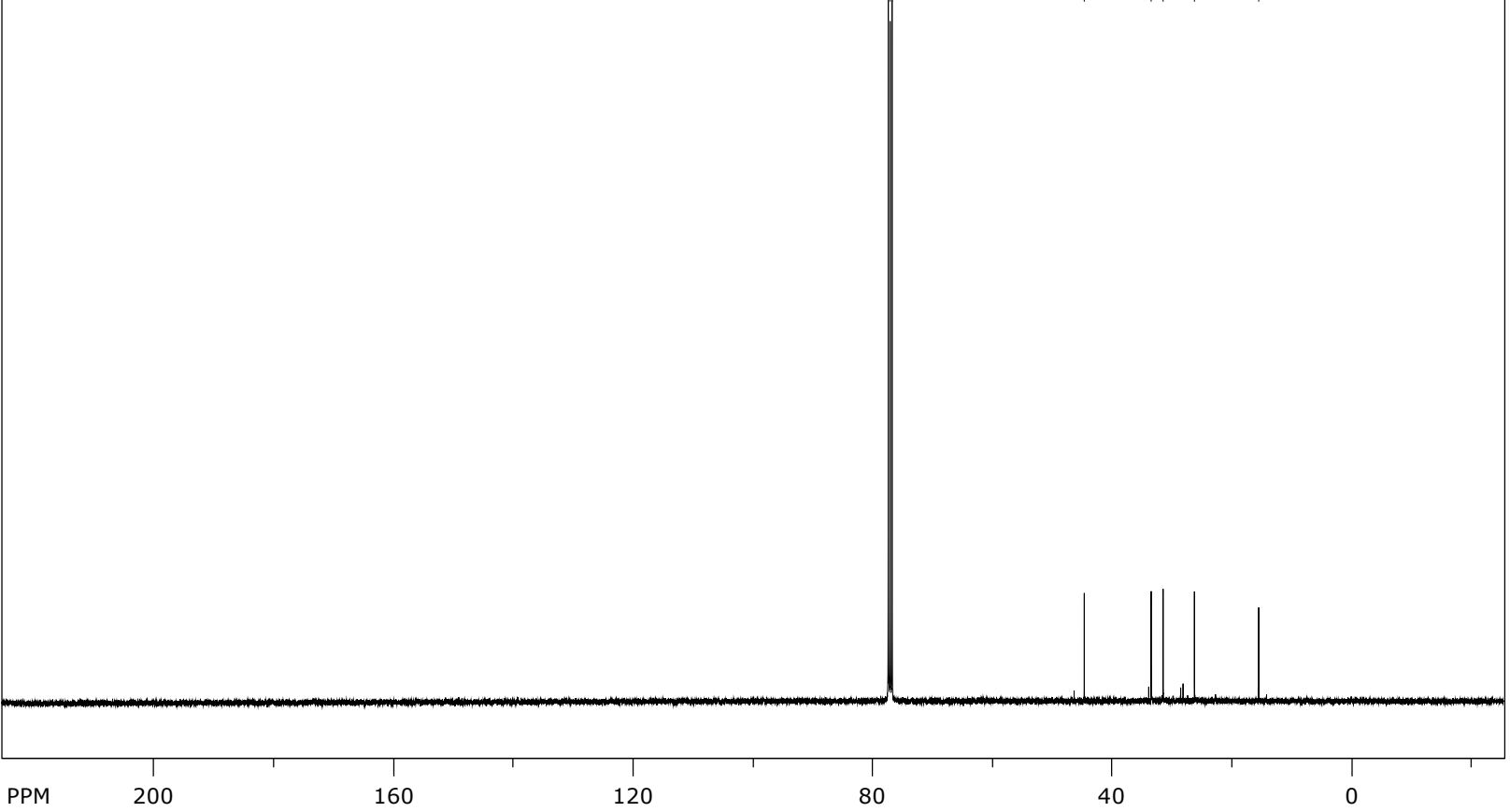

Figure S21: ${ }^{13} \mathrm{C}$ NMR spectrum for 1-chloro-4-(methylthio)butane.

SpinWorks 4: Account SCG10037

Submitted_by D Bates

Sample_name DB236 - pure sample

Lab_number 1.55

Staff/Student ID 201217734

DEPT2 CMR\#

Figure S22 : ${ }^{1} \mathrm{H}$ NMR spectrum for 3. 
SpinWorks 4: Account SCG10037

Submitted_by D Bates

Sample_name DB236 $_{\infty}$ hex recryst 2

Lab_number 1.55

Staff/Student ID $2012 \$ 7734$

DEPT2 CMR\#

( 


\section{References}

(1) Arroyave, F. a.; Richard, C. a.; Reynolds, J. R. Efficient Synthesis of Benzo[1,2-b:6,5-B']Dithiophene4,5-Dione (BDTD) and Its Chemical Transformations into Precursors for $\pi$-Conjugated Materials. Org. Lett. 2012, 14, 6138-6141. https://doi.org/10.1021/ol302704v.

(2) Aradhya, S. V.; Meisner, J. S.; Krikorian, M.; Ahn, S.; Parameswaran, R.; Steigerwald, M. L.; Nuckolls, C.; Venkataraman, L. Dissecting Contact Mechanics from Quantum Interference in Single-Molecule Junctions of Stilbene Derivatives. Nano Lett. 2012, 12 (3), 1643-1647. https://doi.org/10.1021/nl2045815.

(3) Su, T. a; Widawsky, J. R.; Li, H.; Klausen, R. S.; Leighton, J. L.; Steigerwald, M. L.; Venkataraman, L.; Nuckolls, C. Silicon Ring Strain Creates High-Conductance Pathways in Single-Molecule Circuits. J. Am. Chem. Soc. 2013, 135 (49), 18331-18334. https://doi.org/10.1021/ja410656a.

(4) Mészáros, G.; Li, C.; Pobelov, I.; Wandlowski, T. Current Measurements in a Wide Dynamic RangeApplications in Electrochemical Nanotechnology. Nanotechnology 2007, 18 (42), 424004. https://doi.org/10.1088/0957-4484/18/42/424004.

(5) Ferri, N.; Algethami, N.; Vezzoli, A.; Sangtarash, S.; McLaughlin, M.; Sadeghi, H.; Lambert, C. J.; Nichols, R. J.; Higgins, S. J. Hemilabile Ligands as Mechanosensitive Electrode Contacts for Molecular Electronics. Angew. Chemie Int. Ed. 2019, 58 (46), 16583-16589. https://doi.org/10.1002/anie.201906400.

(6) Adak, O.; Rosenthal, E.; Meisner, J.; Andrade, E. F.; Pasupathy, A. N.; Nuckolls, C.; Hybertsen, M. S.; Venkataraman, L. Flicker Noise as a Probe of Electronic Interaction at Metal-Single Molecule Interfaces. Nano Lett. 2015, 15 (6), 4143-4149. https://doi.org/10.1021/acs.nanolett.5b01270.

(7) Diez-Perez, I.; Hihath, J.; Hines, T.; Wang, Z.-S.; Zhou, G.; Müllen, K.; Tao, N. Controlling SingleMolecule Conductance through Lateral Coupling of $\pi$ Orbitals. Nat. Nanotechnol. 2011, 6 (4), 226231. https://doi.org/10.1038/nnano.2011.20.

(8) Soler, J. M.; Artacho, E.; Gale, J. D.; García, A.; Junquera, J.; Ordejón, P.; Sánchez-Portal, D. The SIESTA Method for Ab Initio Order- N Materials Simulation. J. Phys. Condens. Matter 2002, 14 (11), 2745-2779. https://doi.org/10.1088/0953-8984/14/11/302.

(9) Ferrer, J.; Lambert, C. J.; García-Suárez, V. M.; Manrique, D. Z.; Visontai, D.; Oroszlany, L.; Rodríguez-Ferradás, R.; Grace, I.; Bailey, S. W. D.; Gillemot, K.; Sadeghi, H.; Algharagholy, L. A. GOLLUM: A next-Generation Simulation Tool for Electron, Thermal and Spin Transport. New J. Phys. 2014, 16 (9), 093029. https://doi.org/10.1088/1367-2630/16/9/093029.

(10) Sadeghi, H. Theory of Electron, Phonon and Spin Transport in Nanoscale Quantum Devices. Nanotechnology 2018, 29 (37), 373001. https://doi.org/10.1088/1361-6528/aace21.

(11) Dolomanov, O. V.; Bourhis, L. J.; Gildea, R. J.; Howard, J. A. K.; Puschmann, H. OLEX2 : A Complete Structure Solution, Refinement and Analysis Program. J. Appl. Crystallogr. 2009, 42 (2), 339-341. https://doi.org/10.1107/S0021889808042726. 
(12) Sheldrick, G. M. SHELXT - Integrated Space-Group and Crystal-Structure Determination. Acta $\begin{array}{lllllll}\text { Crystallogr. Sect. A } & \text { Found. } & \text { Crystallogr. } & \text { 2015, } & 71 & \text { (1), }\end{array}$ https://doi.org/10.1107/S2053273314026370.

(13) Sheldrick, G. M. Crystal Structure Refinement with SHELXL. Acta Crystallogr. Sect. C Struct. Chem. 2015, 71 (Md), 3-8. https://doi.org/10.1107/S2053229614024218. 